\title{
Motion Rehab 3D Plus: \\ Um Exergame Customizável Aplicado à Reabilitação Física
}

\author{
Mateus Trombetta ${ }^{1}$, Ericles Andrei Bellei ${ }^{2}$, Rafael Rieder ${ }^{1,2}$, \\ Ana Carolina Bertoletti De Marchi ${ }^{1,2,3}$
}

\author{
${ }^{1}$ Curso de Ciência da Computação \\ ${ }^{2}$ Programa de Pós-Graduação em Computação Aplicada \\ ${ }^{3}$ Programa de Pós-Graduação em Envelhecimento Humano \\ Universidade de Passo Fundo (UPF) \\ BR 285 - São José - CEP 99052-900 - Passo Fundo - RS - Brasil \\ $\{132686,168729$, rieder, carolina\} dupf.br
}

\begin{abstract}
Digital games are a motivating factor in physical rehabilitation of individuals. However, most of these games focus on the treatment of a single pathology, which commonly raises the cost of acquiring different solutions. This study presents a customizable $3 D$ exergame, with a personalized activity program, applied in physical rehabilitation of individuals with different pathologies. The game was developed in the game engine Unity. Eleven physiotherapists evaluated the usability and acceptance of the game, through the System Usability Scale and the Technology Acceptance Model, to measure perceived ease of use, and assess behavioral intention. The results were satisfactory, showing that the proposed solution can reduce costs, since it combines all activities into a single product, avoiding the purchase of several games and minimizing learning efforts.
\end{abstract}

Resumo. Jogos digitais são elementos motivadores na reabilitação física de indivíduos. Entretanto, a maioria dos jogos são destinados ao tratamento de uma única patologia, o que comumente eleva o custo com a aquisição de diferentes soluções. Este trabalho tem como objetivo apresentar um exergame $3 D$ customizável, aplicado à reabilitação física de indivíduos com diferentes patologias, com um programa de atividades personalizado. Para o seu desenvolvimento, foi utilizado a game engine Unity. Onze fisioterapeutas avaliaram a usabilidade e a aceitação do jogo, por meio da escala SUS e o modelo TAM, respectivamente. Os resultados foram satisfatórios e permitem concluir que a solução proposta pode reduzir custos, evitando a aquisição vários jogos e minimizando o esforço do aprendizado por parte do profissional.

\section{Introdução}

Nas sessões de fisioterapia convencional, diferentes soluções vêm sendo aplicadas para contribuir e apoiar as reabilitações físicas e cognitivas de indivíduos [Pujari et al. 2017; Turolla et al. 2013; Watanabe et al. 2016]. Os jogos digitais são exemplos de tecnologias interativas recentemente empregadas como ferramentas para reabilitação de pacientes [Fiorin et al. 2014; Junior et al. 2011; Shroeder et al. 2014]. Projetos que envolvem o uso dessas tecnologias consideram o processo de reabilitação exigido, pois é fundamental adequar as atividades interativas em conformidade com as limitações individuais. 
Nesse contexto, [Barros et al. 2013] relatam que o crescente interesse por jogos digitais na reabilitação decorre da possibilidade de captação de movimentos naturais, o que favorece o aspecto motivacional por apresentar um ambiente enriquecido $\mathrm{e}$ diferenciado. Além disso, [Pirovano et al. 2012] ressaltam que os jogos para reabilitação possuem grande potencial, não apenas para auxiliar no processo de reabilitação, mas também para melhorar a manutenção de registros clínicos e futuros métodos de diagnóstico médico. Os autores acrescentam que os dados coletados, associados aos dados do profissional médico, podem ser utilizados para ajustar o nível de dificuldade dos exercícios, avaliar riscos potenciais e aconselhar sobre a terapia.

Entre os jogos utilizados para a reabilitação, estão os exergames, também conhecidos como videogames ativos, que focam no envolvimento do indivíduo por meio de atividades físicas. Com isso, motivam o aprendizado de habilidades motoras e incorporam elementos de competição utilizados nos jogos tradicionais para incentivar a realização dos movimentos propostos [Shapi'i et al. 2015]. A captura dos movimentos no exergame é realizada com o uso de um sensor de movimentos como o Kinect, por exemplo. Esse dispositivo auxilia na percepção dos movimentos naturais do usuário e oferece feedback visual instantaneamente [Bao et al. 2013].

Entretanto, os exergames, em sua maioria, trabalham a reabilitação específica de uma determinada patologia, o que exige do fisioterapeuta a aquisição e o aprendizado de diferentes soluções. Tal necessidade dificulta a incorporação de exergames nas sessões de fisioterapia convencional. Diante disso, este trabalho tem o objetivo de propor um exergame 3D customizável que permite ao fisioterapeuta configurá-lo de acordo com a necessidade da patologia apresentada por seu paciente. $O$ jogo pode possibilitar o uso de uma única solução para diferentes patologias, como no tratamento de pacientes pósAcidente Vascular Encefálico (AVE) ou do equilíbrio em idosos. Para tanto, o jogo auxilia na pré-configuração de atividades $3 \mathrm{D}$ para reabilitação de qualquer tipo de patologia. O jogo também possibilita o controle das informações dos pacientes e das atividades que cada um deve realizar.

Neste artigo, a Seção 2 apresenta a fundamentação teórica. A Seção 3 apresenta as ferramentas utilizadas para a criação do menu configurável e métodos para avaliação do jogo. A Seção 4 aborda o desenvolvimento e funcionamento do jogo. A seção 5 apresenta os resultados e discussões da avaliação do jogo com profissionais de saúde. Por fim, a Seção 6 apresenta as conclusões e os trabalhos futuros.

\section{Fundamentação Teórica}

Há uma grande variedade de estudos relacionados à utilização de exergames em indivíduos em processos de reabilitação, em que a captura dos movimentos é realizada por sensores como o Kinect e o Wiimote. Entre esses estudos, estão os trabalhos de [Norouzi-Gheidari et al. 2013; Passos et al. 2013; Rodrigues and Molz 2014], que utilizam exergames para a reabilitação de pacientes pós-AVE, cada um deles focando em um aspecto físico diferente. Outros trabalhos citam sua utilização na reabilitação de Parkinson [Pachoulakis and Papadopoulos 2016; Palacios-Navarro et al. 2015], Demência [Breton et al. 2012], e em problemas cardiovasculares [Eichhorn et al. 2013].

Apesar desses estudos cumprirem o propósito de auxiliar na terapia convencional e motivar a realização dos exercícios, cada um deles foi desenvolvido em uma ferramenta diferente, o que acaba acarretando dois grandes problemas para o profissional da saúde 
que deseja utilizar esses jogos na clínica. O primeiro está relacionado ao alto custo de aquisição de diversos equipamentos, e o segundo com o aprendizado necessário para utilizar cada um deles. Para tanto, faz-se necessário a criação de um jogo com as seguintes características: (1) Diversidade de atividades: conter diversas atividades para diferentes tipos de patologias, além de facilitar a inserção de novas; (2) Controle de pacientes: possibilitar o cadastro de pacientes para obter informações pessoais e clínicas de todos que estão utilizando o jogo; (3) Menu configurável: para facilitar a busca de atividades e armazenar aquelas que forem selecionadas para cada paciente.

Contudo, para que a configuração seja possível, é preciso armazenar informações de cada atividade disponível no jogo a partir de metadados. Os metadados consistem em registros que possuem um conjunto de atributos ou elementos necessários para descrever um recurso. Por exemplo, um sistema de metadados de uma biblioteca contém um conjunto de registros com elementos que descrevem um livro (e.g., autor, título, data de criação ou publicação) [Wolf et al. 2011]. Esses são descritos com alguma linguagem de marcação, normalmente o XML (Extensible Markup Language), que é um formato para a criação de documentos com dados organizados de forma hierárquica. Entre os padrões de metadados mais utilizados, está o Dublin Core, que é formado por quinze elementos básicos e possui flexibilidade para a inclusão de novos elementos conforme a necessidade de cada usuário [Dublin Core 2017].

Para a abordagem desse trabalho, optou-se pela adaptação do padrão de descrição de atividades de jogos digitais de [Lee et al. 2013]. No trabalho, os autores apontaram 16 elementos principais sobre as informações necessárias à identificação de um jogo. Isso acontece por meio de entrevistas com jogadores, análise de mercado e padrões de buscas. Ressalta-se que, para compor esse padrão, os autores utilizaram elementos do Dublin Core como base. Como o presente trabalho precisa identificar apenas os elementos das atividades e não do jogo como um todo, foram selecionadas apenas os elementos considerados relevantes para esse propósito.

\section{Materiais e Método}

Para a criação do jogo, foi utilizada uma ferramenta de desenvolvimento de jogos Unity 3D e um banco de dados SQLite para o armazenamento de informações sobre os pacientes. A Unity é game engine provida de um conjunto de recursos para o rápido desenvolvimento de aplicações interativas em 3D ou 2D, por meio de linguagem C\# ou JavaScript [Unity Technologies 2017], e que possui suporte a dispositivos como o Kinect. O SQLite é um banco de dados relacional compacto que possibilita ler e gravar dados em um arquivo de disco comum [SQLite 2017], sem a necessidade de servidores para sua utilização. Isso facilita a manipulação durante a utilização do jogo, a integração com a Unity e a ligação entre os pacientes cadastrados e as atividades associadas a eles.

A avaliação do jogo foi realizada com 11 fisioterapeutas, professores do curso de Fisioterapia da Universidade de Passo Fundo (UPF) e da Universidade Regional Integrada do Alto Uruguai e das Missões (URI). Todos os participantes utilizaram a ferramenta a partir de dois casos de uso, conforme detalhado na Tabela 1. Em ambos, foi solicitada a execução das principais funcionalidades do jogo, procurando avaliar compreensão, controle de paciente e organização de atividades. 
Tabela 1. Casos de uso utilizados.

\begin{tabular}{|c|c|}
\hline CASO DE USO 1 & CASO DE USO 2 \\
\hline 1. Inserir um novo paciente com o nome Roberto & 1. Selecionar o paciente Roberto \\
\hline 2. Consultar as informações do paciente Roberto & 2. Remover as atividades de AVE para membros superiores \\
\hline 3. Selecionar o paciente Roberto & 3. Inserir as atividades de Parkinson para membros superiores \\
\hline $\begin{array}{l}\text { 4. Adicionar as atividades de AVE para membros } \\
\text { superiores }\end{array}$ & 4. Iniciar uma das atividades do jogo \\
\hline 5. Iniciar uma das atividades do jogo & 5. Remover o paciente Roberto \\
\hline 6. Encerrar o jogo & 6. Encerrar o jogo \\
\hline
\end{tabular}

Ao término da experimentação, os profissionais responderam dois questionários: Questionário de Avaliação de Aceitação de Tecnologia, construído com base no Modelo de Aceitação de Tecnologia (TAM) proposto por [Davis et al. 1989], e a escala de Usabilidade do Sistema (SUS), proposta por [Brook 1996] para avaliar a usabilidade do jogo. Ambos utilizam a escala Likert de cinco níveis para as respostas.

O TAM é aplicado por pesquisadores para descrever tecnologias de informação após seu uso. Ele composto de 11 questões agrupadas em duas categorias: (1) utilidade percebida, que determina o grau em que se acredita que o uso de uma tecnologia pode melhorar o desempenho e a produtividade, e (2) facilidade de uso, que corresponde ao grau em que se acredita que o uso da tecnologia será livre de esforço, ou seja, fácil de aprender e interagir.

A escala SUS avalia três características: (1) efetividade, que verifica se o usuário consegue completar seus objetivos; (2) eficiência, para identificar o esforço necessário para realização dos testes; e (3) satisfação, que verifica se a experiência foi satisfatória. Ela conta com 10 itens que foram adaptados para o contexto de jogo. O resultado da escala aferida com o SUS é a soma da contribuição individual de cada item. Para os itens ímpares, deve-se subtrair 1 à resposta do usuário, ao passo que para os itens pares, o score é obtido pela subtração da resposta de 5. Depois de obter o score de cada item, somamse os scores e multiplica-se o resultado por 2,5. Dessa forma, o resultado obtido será um índice de satisfação do utilizador que varia de 0 a 100.

\section{O jogo Motion Rehab 3D Plus}

O Motion Rehab 3D Plus é uma evolução do Motion Rehab AVE 3D (software registrado no INPI sob número: BR5120160013737), um exergame destinado à reabilitação motora de membro superior e equilíbrio de indivíduos que sofreram AVE. A versão antiga possui seis atividades em que o paciente deve movimentar as mãos, membros superiores, membros inferiores e tronco, para capturar objetos na tela e pontuar no jogo. Todas as atividades foram pensadas a partir do tratamento convencional da fisioterapia para AVE, o que torna essa versão específica para essa patologia. $\mathrm{O}$ tratamento de outras patologias exige, portanto, o desenvolvimento de novos jogos.

Nessa perspectiva, o Motion Rehab 3D Plus foi desenvolvido com o intuito de permitir a configuração do tratamento, a partir da escolha das atividades pertinentes a patologia sendo tratada. O jogo possibilita ao fisioterapeuta configurar cada sessão de tratamento, personalizando um programa de atividades $3 \mathrm{D}$ específico para cada paciente. 


\subsection{Configuração de Atividades}

A configuração das atividades é a parte essencial para que o jogo cumpra o objetivo de atender a diversas patologias. Com isso, é possível inserir facilmente novas atividades desenvolvidas.

A apresentação das atividades é realizada com a leitura de um arquivo XML que ocorre ao iniciar o jogo e contém todas as atividades disponíveis. Cada atividade possui suas próprias informações, registradas a partir dos metadados, e permite a geração dos blocos visíveis pelo fisioterapeuta para posterior seleção, ilustrados na Figura 1.

Os metadados, adaptados do trabalho de [Lee et al. 2013], incluem informações sobre o desenvolvedor, data de desenvolvimento, número de jogadores, nível de dificuldade, descrição, visualização (primeira ou terceira pessoa) e apresentação (3D ou 2D) de cada atividade. Além disso, foram criadas quatro novas informações para identificar a patologia, o propósito da atividade, o nome do arquivo da imagem e a cena para identificar a atividade a ser iniciada. Essas informações foram necessárias para especificar elementos importantes na busca e descrição de cada atividade durante a execução do jogo.

Conforme ilustrado pela Figura 1, no jogo são apresentadas apenas as informações importantes para a seleção por parte do profissional, sendo: (1) imagem: para representar visualmente o propósito da atividade; (2) descrição textual: para facilitar o entendimento do que deve ser feito no exercício; (3) patologia e propósito: ao qual a atividade se destina; e (4) botão Adicionar: para permitir sua seleção. São gerados blocos diferentes para cada atividade registrada.

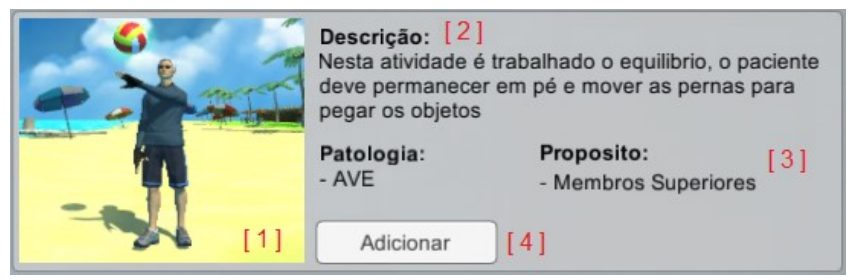

Figura 1. Exemplo da atividade com os dados do XML.

Para a inserção de novas atividades, foram criadas duas telas. A primeira (Figura 2a) contém os metadados que são salvos no arquivo XML. É a partir desses metadados que são gerados os blocos das atividades. Ao cadastrar uma nova atividade, todos os metadados devem ser preenchidos para facilitar a filtragem. A segunda tela (Figura 2b) é utilizada para inserir uma patologia ou propósito que ainda não existe no jogo. De acordo com a Figura 2b, são apresentados os itens já existentes na categoria Propósito, e um campo para digitar a nova categoria que se deseja adicionar. Após isso, o item adicionado é apresentado na tela de inserção de atividades para ser selecionado.

\subsection{Configuração de Atividades}

O jogo foi desenvolvido para ser utilizado por um fisioterapeuta ou qualquer outro profissional da saúde em sessões de reabilitação física. Ele possibilita o controle de informações dos pacientes que utilizam o jogo e também a organização de atividades a serem executadas por cada um. 


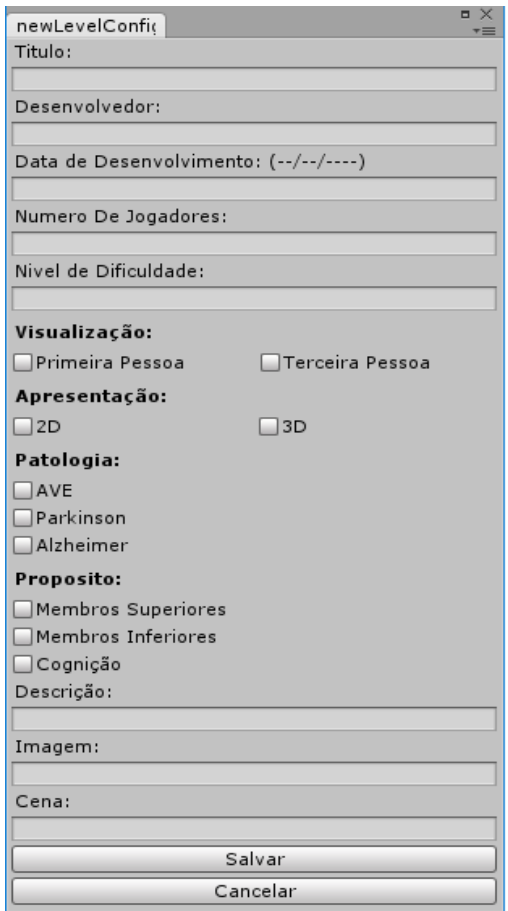

(a)

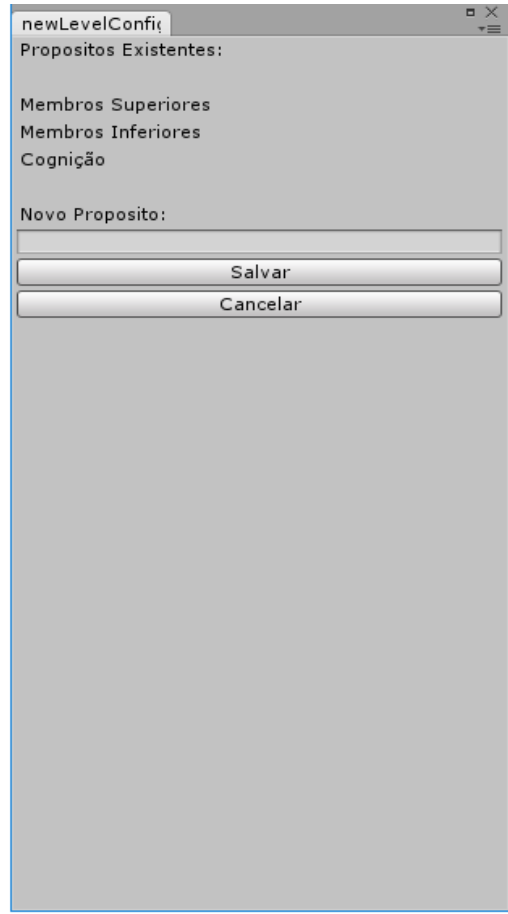

(b)

Figura 2. Telas de (a) inserção de atividades e (b) inserção de categorias.

A tela inicial possui uma interface simples que apresenta o paciente selecionado no canto superior e os quatro principais itens para a configuração do jogo: Iniciar, Selecionar Atividades, Pacientes e Sair. Para melhor compreensão do jogo, o funcionamento será apresentado a partir do menu Pacientes. Ao selecionar a opção Pacientes, o profissional é direcionado para uma tela com as opções de inserir ou selecionar um paciente. No menu de inserção de pacientes, é solicitado um conjunto de informações para o controle de determinado paciente. Essas informações (Figura 3a) foram definidas com a ajuda de um fisioterapeuta para serem abrangentes e atenderem a qualquer patologia. Qualquer informação adicional a ser informada sobre o paciente é registrada no item Observação. A segunda tela é a de seleção do paciente, onde o profissional gerencia todos dados e atividades dos pacientes cadastrados (Figura 3b).

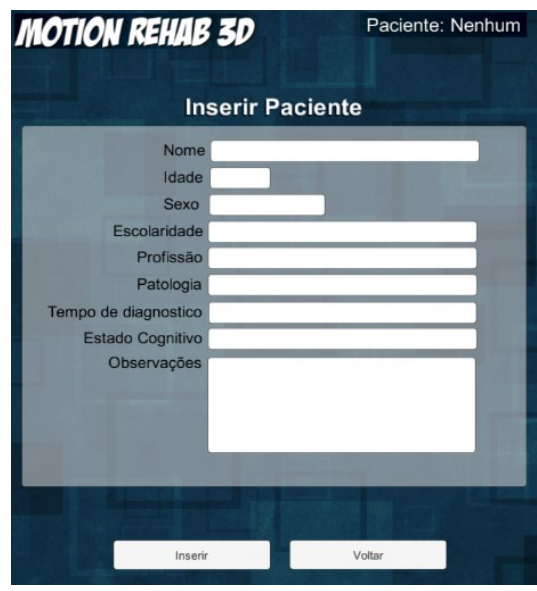

(a)

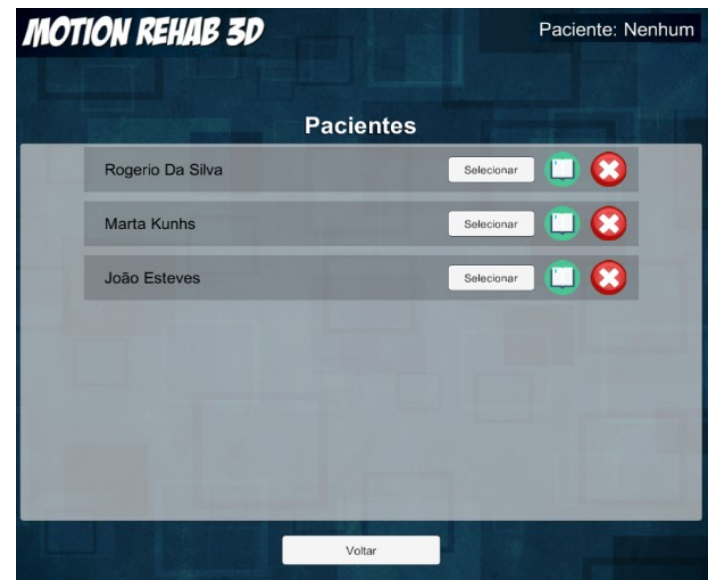

(b)

Figura 3. Telas de (a) inserção de pacientes e (b) seleção de pacientes. 
Com o paciente selecionado, segue-se para a tela de seleção das atividades (Figura 4). Nessa, o fisioterapeuta tem acesso a todas as atividades disponíveis no jogo e pode selecionar aquelas que devem ser realizadas pelo paciente por meio de um mecanismo de filtragem. Como exemplo, se o profissional deseja selecionar atividades para Parkinson voltadas para reabilitação de membros superiores, ele deve marcar a opção "Parkinson" na categoria "Patologia" e a opção "Membro Superior" na categoria "Propósito", fazendo com que o jogo exiba todas as atividades das opções selecionadas.

Para selecionar as atividades, o fisioterapeuta deve clicar no botão "Adicionar". Automaticamente, será apresentado um feedback que a atividade foi selecionada, além de um botão que possibilita sua remoção. Caso nenhum paciente tenha sido selecionado e o profissional clicar no botão de "Adicionar", será apresentada uma mensagem advertindo que é preciso primeiramente selecionar um paciente. Ao voltar para o menu, as atividades selecionadas do paciente são salvas e podem ser acessadas ao abrir o jogo novamente.

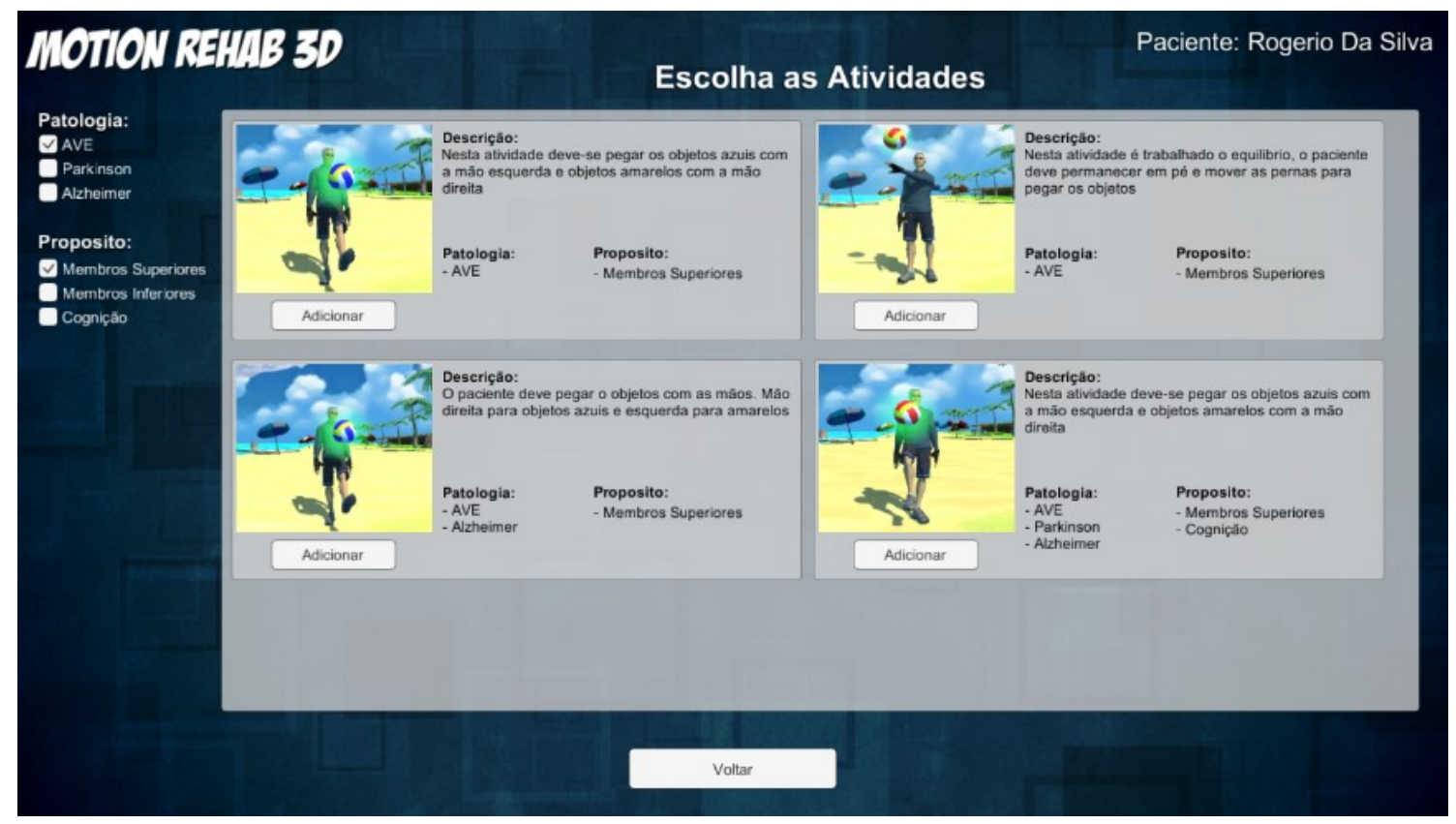

Figura 4. Tela de seleção de atividades.

Com o programa de atividades do paciente definido, o fisioterapeuta pode então ir para a tela de Iniciar o Jogo, onde é possível ter acesso a todas as atividades selecionadas no menu anterior para o paciente. Caso nenhum paciente tenha sido selecionado, a tela exibe um aviso e o botão de selecionar e remover atividades é substituído por um botão de acesso à tela de seleção de pacientes.

As atividades são desenvolvidas em módulos e podem apresentar cenários diferentes, assim como propósitos distintos, como a reabilitação de membros superiores ou inferiores, por exemplo. Além disso, o jogo suporta qualquer tipo de atividade desenvolvida na Unity, possibilitando que qualquer desenvolvedor crie novas atividades, o que facilita sua importação devido ao armazenamento realizado nos arquivos XML. 


\section{Resultados e Discussões}

O jogo proposto obteve, na escala SUS, um índice de satisfação do utilizador final de 90 pontos, classificada como excelente por [Bangor et al. 2009]. Tais evidências indicam que os avaliadores do Motion Rehab 3D Plus se sentiram satisfeitos com o jogo e que, de modo geral, não houve dificuldade com a sua utilização.

Os resultados da escala também permitem averiguar a média dos scores individuais de cada item, conforme apresenta a Figura 5. É importante ressaltar que os scores devem estar acima dos 70 pontos propostos por [Bangor et al. 2009] para serem considerados satisfatórios. Tal regra é válida inclusive para os itens que possuem um aspecto negativo, que é o caso dos itens pares. $\mathrm{O}$ item 2 , por exemplo, apresenta uma média de 90,91, indicando que o jogo não foi considerado complexo. O mesmo raciocínio é usado para os outros itens pares.

Conforme os resultados, o menor score obtido foi no item 10, o que reflete diretamente a necessidade do avaliador em interagir com uma nova tecnologia para reabilitação. Dos 11 avaliadores, apenas 2 já haviam utilizado jogos em suas clínicas, o que exigiu dos demais o aprendizado de uma nova tecnologia para organização de atividades para os pacientes. Acredita-se que esse aprendizado tenha afetado o item 9, diminuindo a confiança na utilização do jogo para um score de 84,09.

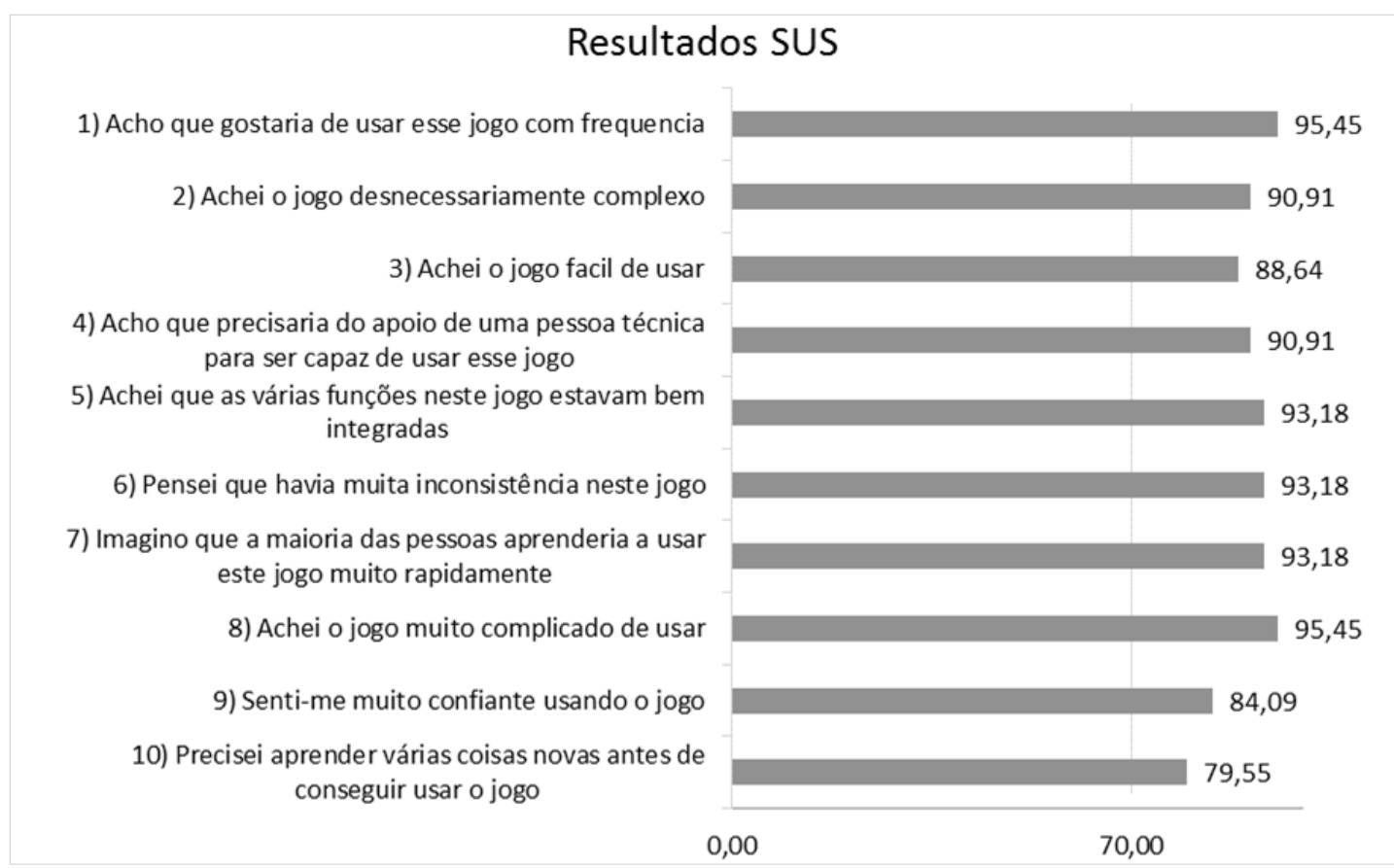

Figura 5. Resultados do questionário sus.

No entanto, pode-se estabelecer outra forma de análise dos resultados a partir do agrupamento dos itens, levando em consideração o trabalho proposto por [Tenório et al. 2011], que relaciona os componentes de qualidade indicados por [Nielsen 2012] e as questões do SUS:

- Eficiência do sistema: relacionados nos itens 5,6 e 8. A média obtida dessas questões foi de 93,93, o que indica que os avaliadores consideraram o jogo capaz para o propósito que se destina. 
- Satisfação dos usuários: representada pelos itens: 1, 4, 9. A média dessas questões foi 90,15 , indicando que os avaliadores gostaram de utilizar o jogo.

- Facilidade de aprendizagem do sistema: representada nos itens 3, 4, 7 e 10. A média dos resultados dessas questões foi de 88.08. Nesse sentido, pode-se concluir que os avaliadores entenderam rapidamente os recursos do jogo.

- Facilidade de memorização: avaliada pela questão 2. O score de 90,91 evidencia que os avaliadores conseguiram fixar as funcionalidades do jogo de maneira satisfatória.

Diferentemente da escala SUS, o modelo TAM avalia a aceitação do jogo a partir de duas categorias e também dos itens individuais de cada uma, utilizando uma média dos valores de 1 a 5 . A Figura 6 apresenta a média dos resultados da categoria utilidade percebida.

\section{Utilidade Percebida}

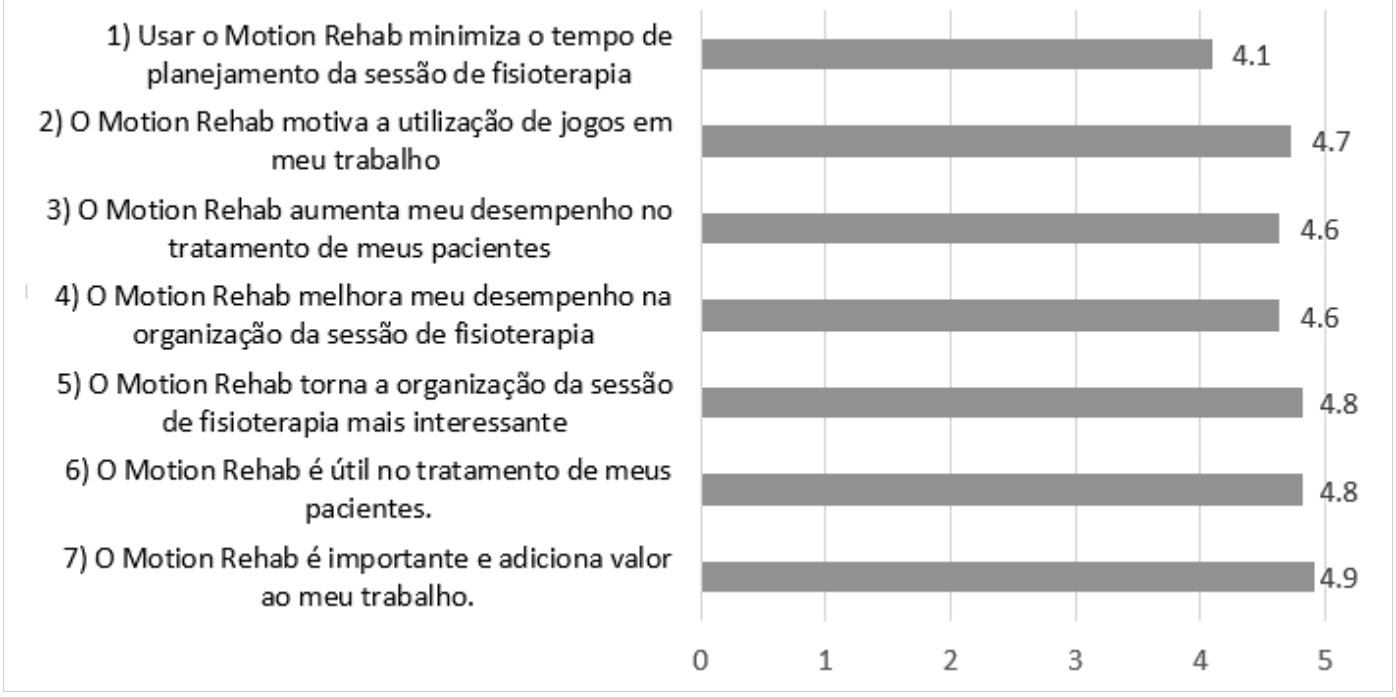

Figura 6. Resultados do teste de utilidade percebida (Modelo TAM).

A média final dessa categoria foi de 4,7, apontando que os fisioterapeutas perceberam a utilidade do jogo. Tal resultado corrobora o resultado do item 1 da escala SUS, em que os avaliadores gostariam de utilizar o jogo com frequência (score $=95,45$ ). A menor média obtida foi no item 1 (média $=4,1$ ). Apesar de ser uma média boa, alguns fisioterapeutas apontaram que esse item não dependia do jogo, pois o planejamento das sessões depende inteiramente do profissional. O jogo foi visto como um facilitador na execução das atividades, o que também pode ser percebido no item 7 (média = 4,9), reforçando que o uso de exergame adiciona valor ao trabalho do profissional.

Os resultados da categoria facilidade de uso estão ilustrados na Figura 7. A média total nessa categoria foi de 4,5. A menor nota obtida foi no item 3 , com uma média de 4,4, que ressalta uma dificuldade acerca da navegação. Acredita-se que houve uma dificuldade para compreender que, para iniciar as atividades, era preciso acessar o menu Jogar, e não o de Atividades. Além disso, é possível observar que a média obtida nessa categoria foi menor que o da utilidade percebida, reforçando os resultados obtidos na facilidade de aprendizagem do jogo na escala SUS. 


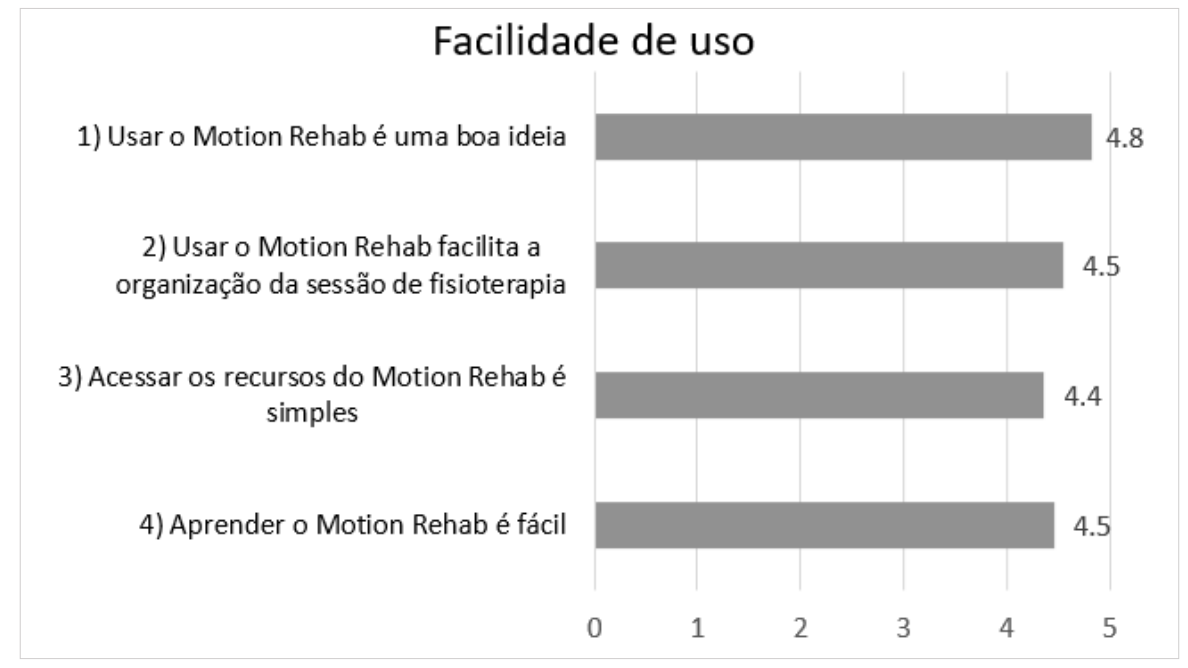

Figura 7. Resultados do teste facilidade de uso (Modelo TAM).

Mesmo com os resultados satisfatórios, foi possível observar alguns elementos que confundiram os profissionais durante a utilização do jogo. $\mathrm{O}$ botão de consultar paciente não apresentava nenhuma informação escrita, apenas uma imagem de um livro, fazendo com que os fisioterapeutas selecionassem o paciente para consultar suas informações sem necessidade. Outro exemplo ocorreu no menu de seleção de atividades, onde não havia certeza se as atividades selecionadas eram salvas ao clicar no botão de voltar para o menu principal. Um dos profissionais indicou a inserção de um botão de salvar para facilitar o entendimento. Ainda nessa tela, foi observado que a filtragem de atividades não estava muito bem destacada em relação as atividades apresentadas, fazendo com que as buscas de atividades fossem realizadas sem a utilização do filtro. Todas os itens que aparentaram problemas foram posteriormente melhorados na interface do jogo. Sobre o contexto do uso do jogo com base nos casos de uso, houve algumas dificuldades nas primeiras interações com o jogo, principalmente no caso de uso 1 . Entretanto, ao realizar o caso de uso 2, os fisioterapeutas já tinham uma grande facilidade na utilização.

\section{Considerações Finais}

A utilização de exergames ganha cada vez mais espaço no tratamento de pacientes, sendo uma técnica eficaz ao mesmo tempo em que promove experiências imersivas e recreativas. Este trabalho apresentou um jogo customizável que auxilia os profissionais de saúde na utilização de exergames em suas clínicas. Espera-se que o jogo possa contribuir com as áreas da Computação e da Saúde, auxiliando na reabilitação de pacientes com algum tipo de patologia. Além disso, a customização do jogo é um fator que pode facilitar a adoção de exergames nas sessões de terapia convencionais. Com o uso de uma solução única, o custo da aquisição e o aprendizado de diferentes exergames são reduzidos.

Nos questionários de avaliação utilizados, foi possível observar questões que dificultaram a utilização do jogo, além de obter sugestões para a melhoria de sua interface. Como trabalhos futuros, pretende-se o desenvolvimento de novas atividades 3D, a inclusão de novas informações sobre as especificações e patologia de cada paciente, e realização de aprimoramentos do jogo conforme as sugestões dos fisioterapeutas. 


\section{Referências}

Bangor, A., Kortum, P. and Miller, J. (2009). Determining What Individual SUS Scores Mean: Adding an Adjective Rating Scale. Journal of Usability Studies. Journal of Usability Studies, v. 4, n. 3, p. 114-123.

Bao, X., Mao, Y., Lin, Q., et al. (5 nov 2013). Mechanism of Kinect-based virtual reality training for motor functional recovery of upper limbs after subacute stroke. Neural Regeneration Research, v. 8, n. 31, p. 2904-13.

Barros, S. L. A., Passos, N. R. S. and Nunes, M. A. S. N. (2013). Estudo inicial sobre Acidente Vascular Cerebral e Serious Games para aplicaçao no projeto "avc" do Núcleo de Tecnologia Assistiva da UFS. Revista GEINTEC: Gestão, Inovação e Tecnologias, v. 3, n. 1, p. 129.

Breton, Z. S. de U., Zapirain, B. G. and Zorrilla, A. M. (oct 2012). KiMentia: Kinect based tool to help cognitive stimulation for individuals with dementia. In 2012 IEEE 14th International Conference on e-Health Networking, Applications and Services (Healthcom).

Brook, J. (1996). SUS: a quick and dirty usability scale. Usability Evaluation in Industry. London: Taylor \& Francis. p. 189-194.

Davis, F. D., Bagozzi, R. P. and Warshaw, P. R. (aug 1989). User Acceptance of Computer Technology: A Comparison of Two Theoretical Models. Management Science, v. 35, n. 8, p. 982-1003.

Dublin Core (2017). DUBLIN core metadata initiative. www.dublincore.org, [accessed on Jan 11].

Eichhorn, S., Koller, V., Schreiber, U., et al. (10 dec 2013). Development of an Exergame for individual rehabilitation of patients with cardiovascular diseases. Australasian Physical \& Engineering Sciences in Medicine, v. 36, n. 4, p. 441-447.

Fiorin, M. R., Marchi, A. C. B. De, Colussi, E. L., Rieder, R. and Trombetta, M. (2014). Motion Rehab: Um jogo sério para idosos com sequelas de Acidente Vascular Encefálico. In Anais do XIV Workshop de Informática Médica. Sociedade Brasileira de Computação.

Junior, R. S. M., Carvalho, R. J. de P., Da Silva, E. B. and Bastos, F. G. (2011). Virtual rehabilitation effect in different Types of treatment. Revista Brasileira de Ciências da Saúde, v. 9, n. 29, p. 56-63.

Lee, J. H., Cho, H., Fox, V. and Perti, A. (2013). User-centered approach in creating a metadata schema for video games and interactive media. In Proceedings of the 13th ACM/IEEE-CS joint conference on Digital libraries - JCDL ' 13.

Nielsen, J. (2012). Usability 101: Introduction to usability. https://www.nngroup.com/articles/usability-101-introduction-to-usability/, [accessed on Feb 11].

Norouzi-Gheidari, N., Levin, M. F., Fung, J. and Archambault, P. (aug 2013). Interactive virtual reality game-based rehabilitation for stroke patients. In 2013 International Conference on Virtual Rehabilitation (ICVR). IEEE. 
Pachoulakis, I. and Papadopoulos, N. (jul 2016). Exergames for Parkinson's Disease patients: The balloon goon game. In 2016 International Conference on Telecommunications and Multimedia (TEMU).

Palacios-Navarro, G., García-Magariño, I. and Ramos-Lorente, P. (12 sep 2015). A Kinect-Based System for Lower Limb Rehabilitation in Parkinson's Disease Patients: a Pilot Study. Journal of Medical Systems, v. 39, n. 9, p. 103.

Passos, N. R. S., Barros, S. L. A., Guimaraes, E. M., et al. (2013). Siirius Surfer: Utilizando jogos serios na reabilitação de tronco para pacientes pós-AVC. In Proceedings of SBGames 2013. Sociedade Brasileira de Computação.

Pirovano, M., Mainetti, R., Baud-Bovy, G., Lanzi, P. L. and Borghese, N. A. (sep 2012). Self-adaptive games for rehabilitation at home. In 2012 IEEE Conference on Computational Intelligence and Games (CIG).

Pujari, A. N., Neilson, R. D., Aphale, S. S. and Cardinale, M. (feb 2017). Upper limb vibration prototype with sports and rehabilitation applications: development, evaluation and preliminary study. Healthcare technology letters, v. 4, n. 1, p. 44-49.

Rodrigues, E. and Molz, R. F. (2014). Physio-DRH: desenvolvimento de game inclusivo para aplicação na recuperação do equilíbrio corporal. In Proceedings of the SBGames 2014. Sociedade Brasileira de Computação.

Shapi'i, A., Bahari, N. N., Arshad, H., Zin, N. A. M. and Mahayuddin, Z. R. (mar 2015). Rehabilitation exercise game model for post-stroke using Microsoft Kinect camera. In 2015 2nd International Conference on Biomedical Engineering (ICoBE).

Shroeder, B., Kunanec, M., Kroese, B., et al. (oct 2014). Rapid recovery: a kayakingbased exergame for shoulder rehabilitation and physical fitness. In 2014 IEEE Games Media Entertainment.

SQLite (2017). About SQLite. https://www.sqlite.org/about.html, [accessed on Nov 11].

Tenório, J. M., Cohrs, F. M., Sdepanian, V. L., Pisa, I. T. and Marin, H. de F. (20 mar 2011). Desenvolvimento e Avaliação de um Protocolo Eletrônico para Atendimento e Monitoramento do Paciente com Doença Celíaca. Revista de Informática Teórica e Aplicada, v. 17, n. 2, p. 210-220.

Turolla, A., Daud Albasini, O. A., Oboe, R., et al. (2013). Haptic-Based Neurorehabilitation in Poststroke Patients: A Feasibility Prospective Multicentre Trial for Robotics Hand Rehabilitation. Computational and Mathematical Methods in Medicine, v. 2013, p. 1-12.

Unity Technologies (2017). Unity3D game engine. http://unity3d.com/unity, [accessed on Nov 11].

Watanabe, T., Endo, S. and Morita, R. (1 dec 2016). Development of a prototype of portable FES rehabilitation system for relearning of gait for hemiplegic subjects. Healthcare Technology Letters, v. 3, n. 4, p. 284-289.

Wolf, A. S., Monteiro, A. P. L. and Valmorbida, W. (2011). Implementation Of The MTD2-BR Metadata Schema To Dspace Tool Seeking The Interoperability With The "Biblioteca Digital Brasileira De Teses E Dissertações (Bdtd)." In $8^{\circ}$ CONTECSI International Conference on Information Systems and Technology Management. 